\title{
Exact solution for infinitely strongly interacting Fermi gases in tight waveguides
}

\author{
Liming Guan, ${ }^{1}$ Shu Chen, ${ }^{1}$, Yupeng Wang, ${ }^{1}$ and Zhong-Qi Ma ${ }^{2}$ \\ ${ }^{1}$ Institute of Physics, Chinese Academy of Sciences, Beijing 100080, China \\ ${ }^{2}$ Institute of High Energy Physics, Chinese Academy of Sciences, Beijing 100049, China
}

(Dated: November 4, 2018)

\begin{abstract}
We present an exact analytical solution of the fundamental systems of quasi-one-dimensional spin-1/2 fermions with infinite repulsion for arbitrary confining potential. The eigenfunctions are constructed by the combination of Girardeau's hard-core contacting boundary condition and group theoretical method which guarantees the obtained states to be simultaneously the eigenstates of $S$ and $S_{z}$ and fulfill the antisymmetry under odd permutation. We show that the total groundstate density profile behaves like the polarized noninteracting fermions, whereas the spin-dependent densities display different properties for different spin configurations. We also discuss the splitting of the ground states for large but finite repulsion.
\end{abstract}

PACS numbers: 03.75.Ss, 05.30.Fk

Introduction. - The experimental progress in manipulating cold atoms in effective one-dimensional (1D) waveguides [1, 2] has stimulated extensive theoretical and experimental study of the 1D strongly correlated atomic systems. Particulary, the experimental realization of Tonks-Girardeau (TG) gases [3, 4] has allowed us to study the fermionization of Bose gas in the strongly interacting limit. More recently an interacting 1D Fermi gas with tunable interaction strengths has also been experimentally realized [5], which offers the opportunity of studying the 1D Fermi gases even in the TG limit. To understand the physical properties of the cold atom in the strongly interacting limit, exact solutions and some refined methods capable of dealing with strong correlations are especially important $[\underline{6}, \mathbf{7}, \mathbf{8}, \underline{9}]$. In the infinitely repulsive limit the many-body state of a TG gas can be constructed via a Bose-Fermi mapping [9]. Despite its long history, the generalization to systems including spin degree of freedom is a highly non-trivial problem and only recently was tackled [10, 11]. Nevertheless the construction of the exact wave function for the fundamental system of indistinguishable spin-1/2 Fermi gas in the TG limit is still lacking despite its great importance [12]. Different from the Bose system whose ground state (GS) is proved to be a degenerated ferromagnetic state [13], the GS of a Fermi system generally falls into the state with lowest total spin value $S[8]$. As we shall clarify later, the mixed symmetry of the spin function renders the generalization of Bose-Fermi mapping to the spin- $1 / 2$ Fermi system difficult and very challenging. In this work we present for the first time an analytically exact solution of quasi-1D Fermi gases with infinite repulsion in trapped potentials.

Model. - We consider a quasi-1d system with N spin$1 / 2$ fermions tightly confined in an elongated potential trap which is described by an effective 1D Hamiltonian

$$
H=\sum_{i=1}^{N}\left[-\frac{\hbar^{2}}{2 m} \frac{\partial^{2}}{\partial x_{i}^{2}}+V\left(x_{i}\right)\right]+g_{1 d} \sum_{i<j} \delta\left(x_{i}-x_{j}\right),
$$

with $g_{1 d}$ being the effective 1D interaction strength [14]. For a harmonic potential, $V\left(x_{i}\right)=\frac{1}{2} m \omega_{x}^{2} x_{i}^{2}$. Despite intensive research [15, 16, 17], there has been rarely rigorous results on the interacting spin- $1 / 2$ fermion systems except the homogenous Yang-Gaudin model [6, 7]. Our exact solution in the strong coupling limit for the fundamental spin- $1 / 2$ Fermi system will provide a firm touchstone for various approximate methods [15, 16, 17] and also deepen our understanding on the few-body system.

Construction of exact ground-state wavefunction. - In general, one can represent the many-body wavefunctions in the space-spin form as $\Psi\left(x_{1}, \sigma_{1} ; \cdots ; x_{N}, \sigma_{N}\right)$. Inspired by the seminal work of Girardeau, the effect of an infinitely strong interaction can be reduced to a hardcore boundary condition

$$
\left.\Psi\left(x_{1}, \sigma_{1} ; \cdots ; x_{N}, \sigma_{N}\right)\right|_{x_{i}=x_{j}, \sigma_{i}=-\sigma_{j}}=0 .
$$

In addition the Pauli exclusion principle enforces the boundary condition $\left.\Psi\left(x_{1}, \sigma_{1} ; \cdots ; x_{N}, \sigma_{N}\right)\right|_{x_{i}=x_{j}, \sigma_{i}=\sigma_{j}}=$ 0 for two particles with the same spins. Therefore the general contacting boundary condition for a TG Fermi gas can be represented as $\Psi\left(x_{i}=x_{j}\right)=0$, which is irrelevant to the spin configurations. Now it is straightforward to observe that the wave function, which is composed of Slater determinant of $N=N_{\uparrow}+N_{\downarrow}$ orthonormal orbitals $\phi_{1}(x), \cdots, \phi_{N}(x)$ occupied by either component of Fermions, fulfills the above boundary condition. Explicitly, we have

$$
\psi_{A}\left(x_{1}, \ldots, x_{N}\right)=(N !)^{-\frac{1}{2}} \operatorname{det}\left[\phi_{j}\left(x_{i}\right)\right]_{i=1, \ldots, N}^{j=1, \ldots, N}
$$

with $\phi_{j}\left(x_{i}\right)$ the eigenstate of the single particle Hamiltonian $H_{i}=-\frac{\hbar^{2}}{2 m} \frac{\partial^{2}}{\partial x_{i}^{2}}+V\left(x_{i}\right)$. So far the spin part of wave function is not considered yet. Since $H$ is spin independent, $H$ is commutable with the total spin operator $\hat{\mathbf{S}}=\sum_{i} \hat{\mathbf{S}}_{i}$, where $\hat{\mathbf{S}}_{i}$ is the spin operator of the ith particle. This implies that the system possesses a global $\mathrm{SU}(2)$ symmetry and the eigenstates of $\mathrm{H}$ are simultaneous eigenstates of $\hat{\mathbf{S}}^{2}$ and $\hat{S}_{z}$. Thus, only the eigenstates 
with the largest eigenvalue $S_{z}=S$ are needed to be considered and the remaining eigenstates can be calculated from them by the lowering operator $\hat{S}_{-}$. In addition, the total wave function of $\mathrm{N}$ indistinguishable fermions has to be antisymmetric under transposition of any two particles.

According to (3), the GS corresponds to the fully filled state with the lowest $N$ orbital occupied and excited states are generated by occupying higher orbitals. Similar to the spinor boson case, the GS is highly degenerate in the TG limit due to the different spin configurations. Among the family of degenerate GSs, the ferromagnetic spin state with $S_{z}=S=N / 2$ is a product of all spins up which is totally symmetric in permutations. The total wave function, antisymmetric under transpositions, takes a factorized form $\Psi=\psi_{A}\left(x_{1}, \cdots, x_{N}\right) \chi_{1}(1) \cdots \chi_{1}(N)$, where $\chi_{1}(i)$ denotes the up-spin and $\chi_{2}(i)$ the down-spin. For the system with fixed up-spin and down-spin particles, the ferromagnetic state with $S_{z}=N / 2-m$ is also totally symmetric in permutations and degenerated with the polarized state, where $m \equiv N_{\downarrow}$ and $n \equiv N_{\uparrow}=N-m$ are the numbers of particles with down-spin and up-spin, respectively. So far, only the ferromagnetic state is constructed. An important issue here is to discuss how the GS degeneracy in the TG limit is split when $g_{1 d}$ is large but finite, or alternatively, to find the GS which could be a good approximation of the true wave function when the interaction strength is very large but not infinite. According to Lieb-Mattis theorem [8], for finite interaction strength, the state with lower $S$ has lower GS energy, therefore the GS for the system with fixed $n$ and $m$ is the state with $S=S_{z}=N / 2-m$. Intuitively, the repulsive interaction term will contribute a positive energy to a state with $S<N / 2$, but it does not contribute to a ferromagnetic state with all spins oriented in the same direction, therefore the Lieb-Mattis theorem seems counter intuitive. One can understand this problem by noticing that a ferromagnetic state with $S=N / 2$ should occupy $\mathrm{N}$ different orbits due to the Pauli principle, whereas for the state with lower $\mathrm{S}$, the particles with opposite spins are allowed to occupy overlapping states and thus lower the energy.

The spin function with $S<N / 2$, described by a Young diagram $[n, m]$, is not totally symmetric. Nevertheless, we can still represent a wave function formally as a product of $\psi_{A}$ and $\psi_{S}$, where $\psi_{S}$ denotes a symmetric function composed of linear combination of product of sign functions and spin functions. Next we shall resort to the group theoretical method to construct $\psi_{S}$.

Before presenting our result, we first introduce some notations of group theory [18]. Let $B_{\alpha}=\left\{b_{1}, b_{2}, \ldots, b_{m}\right\}$ be a set of $m$ different integers where $1 \leq b_{1}<b_{2}<\ldots<$ $b_{m} \leq N$. The $n=N-m$ remaining different integers $a_{1}, a_{2}, \ldots, a_{n}$, satisfying $a_{i} \neq b_{j}$ and $1 \leq a_{1}<a_{2}<$ $\ldots<a_{n} \leq N$ are also determined by the set $B_{\alpha}$. There are $N ! /(m ! n !)$ different sets $B_{\alpha} . b_{j}=n+j$ when $\alpha=1$.
Corresponding to a set $B_{\alpha}$, we define a permutation $P_{\alpha}$,

$$
P_{\alpha}=\left(\begin{array}{cccccccc}
1 & 2 & \ldots & n & n+1 & n+2 & \ldots & N \\
a_{1} & a_{2} & \ldots & a_{n} & b_{1} & b_{2} & \ldots & b_{m}
\end{array}\right) .
$$

Remind that $P_{1}$ is the identical permutation. The left coset of a subgroup $S_{n} \otimes S_{m}$ of $S_{N}$, where $S_{n}$ and $S_{m}$ are respectively the permutation groups of the first $n$ objects and the last $m$ objects, is denoted by $P_{\alpha}\left(S_{n} \otimes S_{m}\right)$. Introduce $Q_{1}=\prod_{i=1}^{n} \prod_{j=n+1}^{N} \operatorname{sgn}\left(x_{i}-\right.$ $\left.x_{j}\right)$, with the sign function $\operatorname{sgn}\left(x_{i}-x_{j}\right)=\left(x_{i}-\right.$ $\left.x_{j}\right) /\left|x_{i}-x_{j}\right|$, and $Q_{\alpha}=P_{\alpha} Q_{1}$. A spin state with $S=S_{z}=N / 2-m$ is denoted by $P_{\alpha} \mathcal{Y}_{1}^{[n, m]} Z_{1}=$ $\mathcal{Y}_{\alpha}^{[n, m]} Z_{\alpha}$, which is a basis tensor of the tensor subspace $\mathcal{Y}_{\alpha}^{[n, m]} \mathcal{T}$ of $S U(2)$ of rank $N$ with the highest weight [18], where $Z_{1}=\chi_{1}(1) \ldots \chi_{1}(n) \chi_{2}(n+1) \ldots \chi_{2}(N)$, $Z_{\alpha}=P_{\alpha} Z_{1}, \mathcal{Y}_{\alpha}^{[n, m]}=P_{\alpha} \mathcal{Y}_{1}^{[n, m]} P_{\alpha}^{-1}$, and $\mathcal{Y}_{1}^{[n, m]}=$ $\left(\sum_{R \in S_{n}} R\right)\left(\sum_{T \in S_{m}} T\right)\left\{\prod_{j=1}^{m}[E-(j n+j)]\right\}$, where $E$ is the identical permutation and $(j n+j)$ is the transposition between $j$ and $n+j$. Our definition for a Young operator [18 coincides with that in 19], but different from that in [20]. The spin states with $S=S_{z}=N / 2-m$ based on one definition are the linear combinations of those on the other.

Theorem. The totally symmetric wave function constructed by the product of the sign functions $Q_{\alpha}$ and the basis tensors $Z_{\alpha}$ is

$$
\begin{aligned}
\psi_{S} & =\left\{\sum_{\alpha=1}^{N ! /(n ! m !)} P_{\alpha}\right\}\left\{Q_{1}\left(\mathcal{Y}_{1}^{[n, m]} Z_{1}\right)\right\} \\
& =\sum_{\alpha=1}^{N ! /(n ! m !)}\left\{\mathcal{Y}_{\alpha}^{[n, m]} Q_{\alpha}\right\} Z_{\alpha} .
\end{aligned}
$$

Proof. Since $Q_{1}$ and $\mathcal{Y}_{1}^{[n, m]} Z_{1}$ both are invariant in left-multiplying by any element of the subgroup $S_{n} \otimes S_{m}$, the action of $\sum_{\alpha} P_{\alpha}$ is proportional to that of the sum over all elements in $S_{N}$ so that $\psi_{S}$ is invariant in $S_{N}$. The last formula in (4) is obtained by rearrangement. $\diamond$

Now it is easy to check that the ground state

$$
\Psi=\psi_{A} \psi_{S}
$$

with $\psi_{A}$ and $\psi_{S}$ given by (3) and (4) fulfills all the requirements of symmetry and hard-core boundary condition and are simultaneously the eigenstates of $\hat{S}^{2}$ and $\hat{S}_{z}$ with $S=S_{z}=\left(N_{\uparrow}-N_{\downarrow}\right) / 2$. As a concrete example, for $N=3$ with $S=1 / 2, \psi_{S}=\sum_{\alpha=1}^{3}\left(3 Q_{\alpha}-1\right) Z_{\alpha}$, where the identity $\sum_{\alpha=1}^{3} Q_{\alpha}=1$ is used for simplification. We note that our constructed exact solution has the same spin structure described by the Young diagram $[n, m]$ as the true wave function when the strength is very strong but not infinite [8]. Therefore, our result is expected to interpolate analytically between the finite-repulsion case and the limit case with infinite repulsion. 


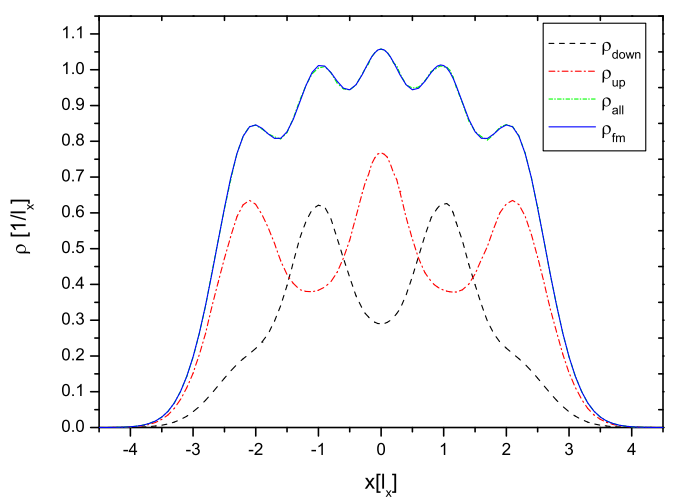

FIG. 1: (color online) The GS density distributions of Fermi gas in the limit of infinite repulsion with $N_{\uparrow}=3$ and $N_{\downarrow}=2$.

Density distributions. - The spin-dependent reduced one-body density matrices are defined as $\rho_{\sigma}\left(x, x^{\prime}\right)=$ $C \int \prod_{i=2}^{N} d x_{i} \Psi^{\dagger}\left(x, X^{\prime}\right) P_{\sigma}^{(1)} \Psi\left(x^{\prime}, X^{\prime}\right)$, where $X^{\prime}=$ $\left(x_{2}, \cdots, x_{N}\right), P_{\uparrow, \downarrow}^{(1)}=\left(1 \pm \hat{\sigma}_{z}^{(1)}\right) / 2$ with $\sigma=\uparrow(\downarrow)$ corresponding to $+(-)$ and $C$ are normalized constants fixed by the conditions $\int d x \rho_{\sigma}(x)=N_{\sigma}$. Here the spindependent single particle densities $\rho_{\sigma}(x)=\rho_{\sigma}(x, x)$ are the diagonal elements of the corresponding reduced density matrices. The total density is defined as $\rho(x)=$ $\rho_{\uparrow}(x)+\rho_{\downarrow}(x)$. After some algebra, we can prove that the total GS density is identical to the density of a polarized $\mathrm{N}$-particle free fermion system which takes the following simple form $\rho(x)=\sum_{l}\left|\phi_{l}(x)\right|^{2}$, where the summation is over the lowest $N=n+m$ single particle states. For the 1D harmonic trap, the orbital are the oscillator eigenstates. In contrast with the ferromagnetic ground state where $\rho_{\sigma}(x)=\left(N_{\sigma} / N\right) \rho(x)$, there is no a simple expression of the spin-dependent density for the general state corresponding to Young diagram $[n, m]$. Nevertheless, one can calculate $\rho_{\sigma}(x)$ directly from the exact ground state wavefunctions. In Fig.1, we display the GS density distributions of a five-particle systems composed of three spin-up and two spin-down fermions. Despite the same total density distributions, the spin-dependent distributions are apparently different from that of the ferromagnetic state. Here the total density profile exhibits five peaks corresponding to that the wavefunction is composed of the lowest five orbitals. However, due to the spin-dependent term of eq. (4), the spin-dependent density profiles are reorganized so that the spin-up part and spin-down part avoid overlapping together and show alternative peak structure to lower the energy. Although the exact many-body wave function is constructed, the calculation of the density distribution and momentum distribution for a large system remains a difficult task due to the time consuming to calculate multidimensional integrals. Nevertheless, some robust features are found

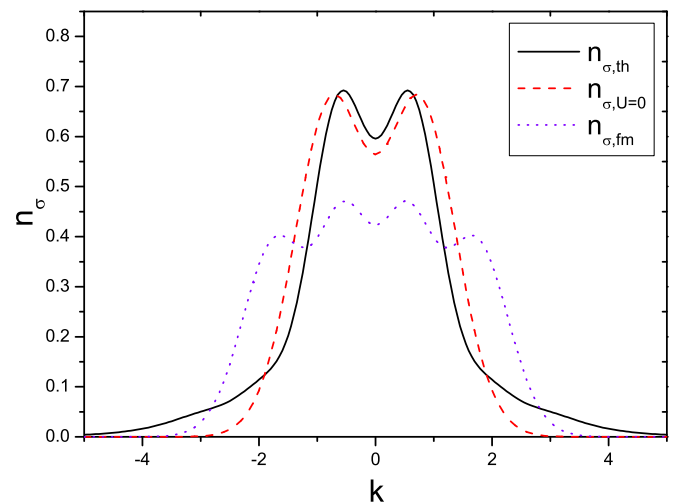

FIG. 2: (color online) The GS momentum distributions $n_{\sigma}(k)$ for the system with $N_{\uparrow}=N_{\downarrow}=2$. Solid line corresponds to spin singlet, dotted line to ferromagnetic state and dashed line to the case of free fermion for comparison.

to be not sensitive to the size. For example, the peaks in the spin-up and spin-down density distributions appear alternatively and the density distributions also show the parity symmetry.

The momentum distribution can be directly obtained from the Fourier transformation of the reduced density matrices $n_{\sigma}(k)=(2 \pi)^{-1} \int d x d x^{\prime} e^{i k\left(x-x^{\prime}\right)} \rho_{\sigma}\left(x, x^{\prime}\right)$. For the ferromagnetic ground state, we have $n_{\sigma}(k)=$ $\left(N_{\sigma} / N\right) \sum_{l=0}^{N-1}\left|\widetilde{\phi}_{l}(k)\right|^{2}$ with $\widetilde{\phi}_{l}(k)$ the Fourier transformation of the $l$-th oscillator eigenstate. Similarly, such a simple expression does not generally hold true for the spin state with the Young diagram $[n, m]$. As shown in Fig.2, the momentum distribution displays quite different behavior for the spin singlet and ferromagnetic state. Comparing with the noninteracting case of $g_{1 d}=0$ where $n_{\sigma}(k)=\sum_{l=0}^{N_{\sigma}-1}\left|\widetilde{\phi}_{l}(k)\right|^{2}$, the momentum distribution develops a wide tail.

Comparison with system with large but finite repulsion.- Apart from infinite repulsion limit, there is no analytical solution available for the harmonic potential. However, for the small particle system, we can apply the exact diagonalization method 21] to calculate its GS properties and compare with our analytical result in the infinite limit. In terms of the fermionic creation and destruction operators $a_{i \sigma}^{\dagger}$ and $a_{i \sigma}$ of the axial harmonic oscillator, we get the many-body Hamiltonian corresponding to (11)

$$
H=\hbar \omega_{x} \sum_{i, \sigma}\left(i+\frac{1}{2}\right) \hat{a}_{i \sigma}^{\dagger} \hat{a}_{i \sigma}+U \sum_{i j k l} I_{i j k l} \hat{a}_{i \uparrow}^{\dagger} \hat{a}_{j \downarrow}^{\dagger} \hat{a}_{k \downarrow} \hat{a}_{l \uparrow},
$$

where $I_{i j k l}=l_{x} \int_{-\infty}^{\infty} d x \phi_{i}(x) \phi_{j}(x) \phi_{k}(x) \phi_{l}(x)$ are the dimensionless interaction integrals with $l_{x}=\sqrt{\hbar / m \omega_{x}}$ and $U=g_{1 d} / l_{x}$. The Hamiltonian (6) can be exactly diagonalized in the truncated basis of eigenstates of the har- 

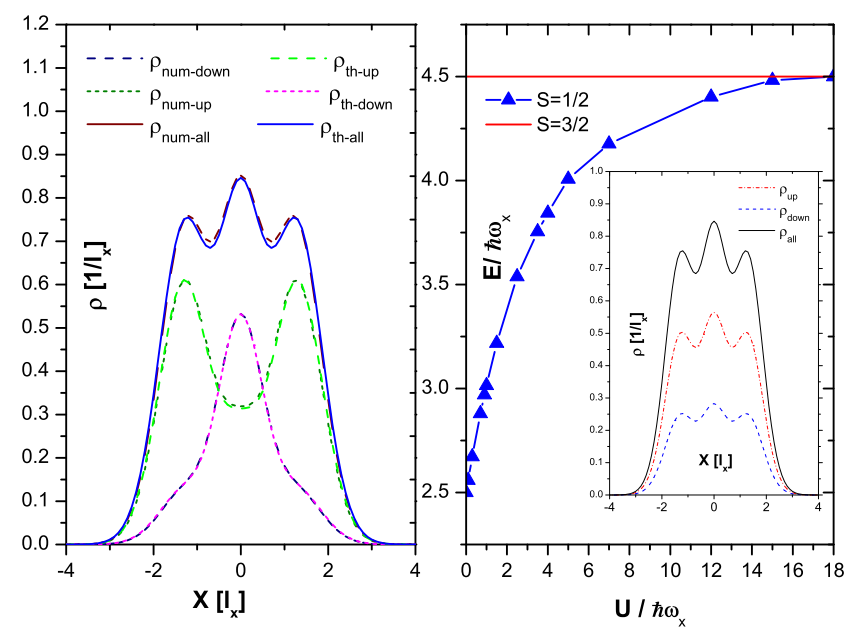

FIG. 3: (color online) (a) Comparison of GS density distributions obtained by ED for the system with $N_{\uparrow}=2$ and $N_{\downarrow}=1$ with the analytical result of $S=1 / 2$ state. (b) The energy versus the interaction for different spin state. The density distribution for the ferromagnetic state (inset).

monic oscillator and then the GS density can be calculated numerically. Fig. Ba shows the 3-particle state corresponding to the Young diagram $[2,1]$, compared to the results obtained from exact diagonalization (ED) of (6) with parameter $U=15 \hbar \omega_{x}$. The numerical results are in perfect agreement with our exact results, which indicates that the limit of infinite repulsion is practically reached at $U=15 \hbar \omega_{x}$ 21, 22]. Instead, as displayed in the inset of Fig. 3 b, the spin-dependent distribution for the degenerate ferromagnetic GS state displays quite different behaviors from the true GS of the system with large finite repulsion. We also show how the GS energy of the state with $S=1 / 2$ changes versus the increase of interaction strength in Fig. 3 b. In the large repulsion limit, it tends to become degenerate with the ferromagnetic state. It would be interesting to compare our results with Ref. [23], where the stability of Fermi gases with the presence of attractive p-wave interaction has been discussed. In the limit case with absence of p-wave interaction, its phase diagram is consistent with our conclusion, i.e., the GS is an antiferromagnetic state.

Experimental realizability and detections. - As the Feshbach resonances in qausi-1D 2-component Fermi system have been observed [5], in principle the 2-component fermionic TG gas can be realized. For the highdimensional system, the physics in the infinitely interaction limit (the unitary limit) as well as the BEC and BCS crossover around the unitary limit have been studied extensively. The main challenge for realization of quasi-1D Fermi system comes from that both the Fermi energy and temperature should be much lower than the transverse confining energy. The spin-dependent density distribution might be detected within the current techniques which allows for measurement of specie-dependent properties 24.

Summary. - We have constructed the exact eigenstates of the fundamental system of quasi-1D spin- $1 / 2$ fermions with infinite $\delta$ repulsion by means of group theoretical method. While the infinite repulsion is described by a hard-core boundary condition, the group theoretical construction guarantees our wave function automatically fulfilling the permutation symmetry and being the eigenstates of $S$ and $S_{z}$. The construction scheme and the formula for spin densities are valid independent of the trapping potential and the particle number. For large but finite repulsion we have calculated the ground state for a few-particle system numerically by using the exact diagonalization method. The numerical result is found to be in excellent agreement with our analytical result. Our construction of exact eigenstates is valid even when a Zeeman term in the Hamiltonian exists because it does not change the nature of the states.

This work is supported by NSF of China Nos. 10821403, 10574150, 10675050 and National Program for Basic Research of MOST, China.

* Electronic address: schen@aphy.iphy.ac.cn

[1] A. Görlitz, et.al., Phys. Rev. Lett. 87, 130402 (2001).

[2] H. Moritz, et.al., Phys. Rev. Lett. 91, 250402 (2003); T. Stöferle, et.al., ibid. 92, 130403 (2004).

[3] B. Paredes, et. al., Nature 429, 277 (2004).

[4] T. Kinoshita, et. al., Science 305, 1125 (2004).

[5] H. Moritz, et. al., Phys. Rev. Lett. 94, 210401 (2005).

[6] C. N. Yang, Phys. Rev. Lett. 19, 1312 (1967).

[7] M. Gaudin, Phys. Lett. 24A, 55 (1967).

[8] E. H. Lieb and D. Mattis, Phys. Rev. 125, 164 (1965).

[9] M. D. Girardeau, J. Math. Phys. (N.Y.) 1, 516 (1960).

[10] M. D. Girardeau and A. Minguizzi, Phys. Rev. Lett. 99, 230402 (2007).

[11] F. Deuretzbacher, et. al., Phys. Rev. Lett. 100, 160405 (2008)

[12] We note that 'Model II' in Ref. 10] actually deals with the distinguishable 2-component system.

[13] E. Eisenberg, et. al., Phys. Rev. Lett. 89, 220403 (2002); K. Yang, et. al., Int. J. Mod. Phys. B 17, 1027 (2003).

[14] M. Olshanii, Phys. Rev. Lett. 81, 938 (1998).

[15] A. Recati, et. al., Phys. Rev. Lett. 90, 020401 (2003).

[16] G. E. Astrakharchik, et. al., Phys. Rev. Lett. 93, 050402 (2004); L. Kecke, et. al., ibid, 94, 176802 (2005).

[17] Gao Xianlong, et. al., Phys. Rev. A 73, 033609 (2006).

[18] Z.-Q. Ma, Group Theory for Physicists, (World Scientific Press, Singapore, 2007).

[19] H. Boerner, Representations of Groups, (North-Holland, Amsterdam, 1963).

[20] M. Hamermesh, Group Theory, (Addison-Wesley, Massachusetts, 1962)

[21] F. Deuretzbacher, et al., Phys. Rev. A 75, 013614 (2007).

[22] Y. Hao, et. al., Phys. Rev. A. 73, 063617 (2006); S. 
Zöllner, et. al., Phys. Rev. A 74, 063611 (2006).

[23] A. del Campo, et al., Phys. Rev. A 76, 013615 (2007).

[24] M. W. Zwierlein, et al., Science 311, 492 (2006); G. B.
Partridge, et al., Science 311, 503 (2006). 